\title{
Towards a practice-based view of Information Systems Resilience Using the Lens of Critical Realism
}

\author{
Amitrajit Sarkar \\ University of Canterbury, \\ NZ \\ Amit.Sarkar@ara.ac.nz
}

\author{
Stephen Wingreen \\ University of \\ Canterbury, NZ \\ stephen.wingreen@can \\ terbury.ac.nz
}

\author{
John Ascroft \\ Jade Software \\ Corporation, NZ \\ jascroft@jadeworld.com
}

\author{
Ravishankar Sharma \\ University of Canterbury, NZ \\ rs.sharma@canterbury.ac.nz
}

\begin{abstract}
Disasters, natural or otherwise, are not rare events and organizations must develop resilience as a governance mechanism for business continuity, growth, and sustainability. It is critical for organizations not only to survive after a disaster but also to bounce back. Organizational resilience has gained upward attention in recent years. This research focuses on an aspect of organizational resilience, i.e., on Information Systems (IS) resilience. This study focuses on understanding the decision making process of senior executives in context to IS resilience in large organizations. Authors present an in-depth case study of a large New Zealand organization adapting with the aftermath of crisis, as well as the lessons they learned along the way. The case study vividly follows dramaturgical guidelines as prescribed by Myers and Newman. The paper shares some important lessons learned by the organization and also proposes a model for IS resilience planning and decision making in light of a strategy-implementation bicycle and causal model to understand decisionmakers' perspective to understand decision priorities.
\end{abstract}

\section{Introduction}

Disasters happen regularly, and cause adversity. Global warming is increasing the risk of extreme weather conditions. Hurricane Katrina in 2005, the Great East Japan earthquake of 2011, the Christchurch earthquakes in 2010 and 2011, and more recently floods in Mozambique each posed unique challenges both to society and commerce. It is critical for the organizations not only to survive after a disaster but also to bounce back. Organizational resilience has become more important to both academics and practitioners in recent years as a dimension of firm governance. Information Systems (IS) resilience is the aspect of organizational resilience that focuses on the firm's information systems. To the best of the researchers' knowledge, there are no theories of IS resilience, and no research has been undertaken to understand the decision making process of senior executives in the context of IS resilience in large organizations.

To a large extent, most organizations are dependent on complex Information Systems (IS) and digital platforms to manage their businesses, which require IS to operate reliably under a variety of adverse circumstances and crisis situations. In the context of delivering services in a crisis situation, IS are considered to be the most susceptible components of the organization, because interruptions affect entire organizational ecosystems which then suffer from the disruption and its related effects [1]. One crucial need for organizational resilience research is to examine how firms achieve the continuance of stable and reliable IS services under a range of adverse operating conditions. To our knowledge, there has been no systematic examination on how IS resilience planning decisions are made, or the role of IS resilience in firm governance. Prior research has addressed disaster recovery (DR), business continuity planning (BCP) and other related issues and mostly focused on strategic IS planning, particularly developing best practice for it (Hann and Weber, 1996). This stream of research has resulted in the development of high level IT governance models, rather than inspecting previous disruptions to determine the finer details of what really happened, how to prevent a recurrence, and ensure IS resilience [2].

We see three problems with the prior research. First, it is mainly prescriptive in nature [3] [4]. Second, there is evidence of a disconnect between what scholars say organizations should be doing with respect to IS planning practices and what decision makers in organizations are actually doing and why they are doing so [3][4]. Third, although research has proposed highlevel IT governance models, the models have not been validated in adverse or crisis circumstances to see if they actually represent how firms respond to crisis [3][4]. To our knowledge, there is a gap with regard to these three 
afore mentioned limitations, so more research is needed to extend and apply IT governance concepts to IS resilience. The primary contribution of this research will be to address this gap by proposing both a conceptual IS resilience strategy framework and a model for how decision makers plan for IS resilience.

IS resilience is comprised of a complex structure and process of decision making which includes alignment between IT and business strategies, improved focus on IT investment for strategic priorities, avoidance of potential business risks, and capitalization on current business opportunities. So, IS resilience encompasses a variety of IT decision types. While some decisions have a clear strategic orientation, others may address strategic and business related objectives, and the rest may lie somewhere in between. Also, an IS resilience plan is unique with respect to other types of plans because an IS resilience plan is intended to be implemented and executed during a time of a crisis situation, when there is a high degree of uncertainty and ambiguity. In theory, IS resilience should be aligned with the overall organizational strategy, and therefore fall under the wider umbrella of organizational resilience. Increasingly, IT governance receives a lot of attention from both scholars and practitioners as the advantages of IT governance are being recognized [5] [6]. Peter Weill's IT governance framework explains how decision rights and responsibilities are distributed within the IS function in organizations, by his definitions of IT archetypes and IT domains, but it does not elucidate why decision rights and responsibilities are distributed the way they are. Weill's definition of an IT archetype encompasses the type of person who has decision rights, and the IT domain includes the decision responsibilities of each IT functional area, but both were developed only in the context of IT governance, not IS resilience [5]. It is therefore the goal of this research to develop and validate an IT governance framework in the context of IS resilience that will account for how decisions are made by senior executives; specifically, this study aims to examine how senior executives make decisions to ensure IS resilience. Toward this goal, we have chosen to investigate Jade Software Corporation because it is an exemplar of the theoretical concepts and executive behavior that we would expect in the context of IS resilience. During the course of this study, Jade was actively involved in the domain of IS resilience planning, prioritization, and alignment as the result of a major crisis, the Christchurch earthquakes of 2011. Because of this, we expected Jade to be deeply engaged with IS resilience, and that we would observe a full, rich range of IS resilience planning and decisionmaking.
We adopt the case study method to develop an initial model of the domain of IS resilience planning, since the case study approach is appropriate for situations where research is in its early, formative stages and not supported by a strong theoretical base. Case studies are necessary for the production of theoretical exemplars, which is in turn a prerequisite for the development of good theories and a healthy discipline [7] [8]. Case studies are suitable for research objectives of an explanatory nature, which attempt to answer why and how questions that focus on contemporary events [9]. Using interviews, direct observation, and archival data, we construct causal maps of the IS resilience planning and decision-making domain. The resulting causal map is developed and validated from in-depth interviews of the executives involved in the IS resilience planning and decision-making process. This approach provides a means of obtaining the insights of practicing managers to understand the issues related to IS resilience planning in a large organization. The resulting theory will be a faithful representation of IS resilience planning as it occurs "in the wild", rather than the closed confines of the theorist's study.

This paper is structured as follows. First, the literature on IS resilience, IT governance, IS resilience planning, and IT governance framework is reviewed. A general description of the Jade Software case will be provided in the context of its activities to cope with the aftermath of a major earthquake. The paper then describes the research methodology, in which the case study method is employed to determine how senior executives at Jade implement their decision priorities in order to ensure IS resilience. Detailed analysis of interviews with the executive management team are employed to enrich our interpretation of the case study. The paper concludes with the discussion of the resulting IS resilience strategy framework and decision-making model. We also discuss the relevance of this research for both practitioners and academics and we propose some recommendations for further research in the area of IS resilience.

\section{Literature Review}

\subsection{IS Resilience}

The concept of resilience has been a prominent and emerging topic in various scientific fields, however, as resilience research encompasses a wide range of disciplines such as ecology, psychology and engineering, as well as different research contexts and topics, it is not surprising that the concept lacks an accepted common definition across disciplines [10]. 
Organizational resilience, however, has been studied extensively by researchers [11]. After an extensive literature review, we were able to find only one paper [12] on the topic of IS resilience in the context of developing countries. Heeks and Ospina, also had difficulty finding a clear definition of IS resilience in previous literature, and proposed nine attributes of IS resilience for the context of developing countries: robustness, self-organization, learning, redundancy, rapidity, scale, diversity and flexibility, and equality [12]. Because our research was executed in the context of executive planning and decision making for IS resilience, we have adapted six attributes as identified by McManus for our definition in the context of IS resilience, namely overall situation awareness, decreased vulnerabilities, increased adaptability, risk intelligence, flexibility and agility. These terms are defined in Table 1 [13]. It is noteworthy that there are correspondences between McManus' attributes and those of Heeks and Ospina.

Table 1.Attributes of IS Resilience

\begin{tabular}{|l|l|l|}
\hline $\begin{array}{l}\text { IS } \\
\text { Resilience } \\
\text { Stages }\end{array}$ & $\begin{array}{l}\text { Set of } \\
\text { Attributes }\end{array}$ & Definition \\
\hline Anticipation & $\begin{array}{l}\text { Situation } \\
\text { awareness }\end{array}$ & $\begin{array}{l}\text { The ability to identify } \\
\text { and understand changes } \\
\text { in the environment. }\end{array}$ \\
\hline Anticipation & $\begin{array}{l}\text { Risk } \\
\text { intelligence }\end{array}$ & $\begin{array}{l}\text { The ability to identify } \\
\text { and anticipate risks. }\end{array}$ \\
\hline Coping & $\begin{array}{l}\text { Management } \\
\text { of } \\
\text { vulnerabilities }\end{array}$ & $\begin{array}{l}\text { The capability to deal } \\
\text { with major } \\
\text { vulnerabilities. }\end{array}$ \\
\hline Adaptation & $\begin{array}{l}\text { Adaptive } \\
\text { capacity }\end{array}$ & $\begin{array}{l}\text { The capability to } \\
\text { respond to and adapt to } \\
\text { the changing } \\
\text { environment. }\end{array}$ \\
\hline Adaptation & Flexible & The ability to change. \\
\hline Adaptation & Agile & $\begin{array}{l}\text { The ability to produce } \\
\text { timely responses to } \\
\text { changing environments } \\
\text { and conditions. }\end{array}$ \\
\hline
\end{tabular}

A definition of IS resilience is introduced based on these characteristics for the purpose of our study. It may be defined as:

IS resilience is a function of an organization's overall situation awareness related to IS, management of IS vulnerabilities, and adaptive capacity, risk intelligence, flexibility and agility of IS in a complex, dynamic, and interconnected environment.
It is worth mentioning, this conceptualization distinguishes the stages of resilience (1) anticipation, (2) coping, and (3) adaptation (see Table 1).

Traditionally the definition of resilience focuses on an event-based approach that identifies potential risks and prepares response measures for each of them, whereas, our definition of IS resilience proposes a process based approach whose goal is to build a sustainable business model. The process based approach embeds resilience-thinking in the culture of an organization, which differentiates it from simply suggesting a corrective measure for a particular event [11]. In other words, our definition of IS resilience is about the planning and implementation of resilient systems, not merely the attributes of resilient systems.

\subsection{Peter Weill's IT Governance Framework}

IT governance is a vital issue in context to IS resilience, since IS resilience by definition is a subdomain of IT governance. While there are many definitions of IT governance, the following two definitions are widely used in IS research.

IT governance is the responsibility of the Board of Directors and executive management. It is an integral part of enterprise governance and consists of the leadership and organizational structures and processes that ensure that the organization's IT sustains and extends the organization's strategy and objectives (IT governance Institute, 2001).

IT governance is the organizational capacity exercised by the Board, executive management and IT management to control the formulation and implementation of IT strategy and in this way ensures the fusion of business and IT [14].

These definitions emphasize the same aspects: alignment of business and IT, and the primary responsibility of the board and senior executives. Van Grembergen's definition also specifies that IT management must participate in IT governance processes. It is important to note that there is a clear distinction between IT management and IT governance. IT management is engrossed in the effective management of IT operations and supply of IT resources, whereas, IT governance is a much larger concept and focusses on performance and transformation of IT to meet present and future demands of the business and its customers [14]. 
Peter Weill's IT governance framework describes how decision rights and responsibilities are spread within the IT function in organizations, by his definitions of IT archetypes and IT domains, but it does not elucidate why decision rights and responsibilities are distributed the way they are or how the decision makers make decisions. In our research we will answer how senior executives make decisions in time of crisis to ensure IS resilience, which will be a valuable contribution to the existing body of knowledge. Weill's definition of an IT archetype involves the type of professional who has decision rights, and the IT domain comprises the decision responsibilities of each functional IT area [5]. Decision rights indicate a decision-maker with knowledge needed to make those decisions, since a decision right specifies who in a firm has the authority to make what decisions. Decision rights essentially move to the department where the relevant knowledge resides ("delegation" solution), or the relevant knowledge must be moved to the locus of decision rights ("transmission" solution) [15]. Weill implicitly assumes that there should be alignment of decision makers' interests with the strategic interests of the firm. According to Weill, IT governance is not explicit decisions about IT, but about who makes what decisions, who has input and how the decision makers are held accountable for those decisions. IT governance encompasses five major decision domains. First, IT principles comprise the high-level decisions about the strategic role of IT in the business. Second, IT architecture includes an integrated set of technical choices to guide the organization in satisfying business needs. Third, IT infrastructure consists of the centrally coordinated, shared IT services that provide the foundation for the enterprise's IT capability. Fourth, business application needs are the business necessities for purchased or internally developed IT applications. Last, prioritization and investment decisions determine how much and where to invest in IT. Also, there are six archetypal approaches to IT decision making, ranging from highly centralize to highly decentralize. According to Weill, most enterprises employ a variety of approaches, using different approaches for different decisions [5].

Currently, there is an inundation of IT management frameworks and standards, each catering to a narrow silo in the firm. A general lack of clarity still exists, when it comes to what constitutes an overarching IT governance framework focused specifically on the senior management's role. IT governance, the term defined as "specifying the decision rights and accountability framework to encourage desirable behavior in the use of IT" [5], constitutes the most universal and systematic approach to solving the business problems associated with IT in the organizational context.

\subsection{IS Resilience Planning}

IS planning plays a crucial role in today's complex, connected, unpredictable, and dynamic corporate world. IT is incorporated into all aspects of business operations and the need for strategic IS planning is of great importance in achieving success. It is defined as the process of strategic thinking that identifies the most required IS on which the organization can implement and impose its long-term IS activities and policies [4]. Earl stated that IS planning is a mixture of formal activities and informal behavior. It may be either a special effort or part of overall organizational planning. However, relatively few organizations successfully adapt to the demands of constant change by the strategic use of IS [3].

Prior studies of IS planning practices in organizations indicate that varied differences exist. Organizations differ in terms of how much IS planning they do, the IS planning methodologies they use, the employees involved in IS planning, the alignment between IT and the business, the focus of IS plans, and the ways in which IS plans are implemented [4]. IS planning has been used to accomplish three major objectives: (1) recognizing organizational opportunities and problems where IS might be used successfully; (2) identifying the resources required for IS to be applied successfully to these problems and opportunities; and (3) developing strategies and processes to allow IS to be applied successfully to these opportunities and problems [4]. Thus, the IS planning process is recognized as an exercise to improve an organization's strategic alignment with business-IT objectives; to meet shortterm and long-term organizational needs; and to provide the ability to create impact through competitive advantage.

The goals of IS planning include improving systems' architecture, infrastructure capability and reliability from IS/IT investments; managing information resources effectively; and securing user satisfaction. However, IS resilience planning is unique with respect to other types of plans because an IS resilience plan anticipates that at least some elements of the plan will be implemented during a time of crisis or adverse circumstances, when there is a high degree of uncertainty. Moreover, if decision rights are not delegated to be exercised in the presence of high uncertainty, organizations may not be able to respond quickly enough to the IS prospects and problems they meet. 
IS resilience shares some commonality with crisis management. Crisis management is the process by which an organization deals with any major unpredictable event threatening to harm the organization, its stakeholders, as well as its customers and suppliers. Vargo and Seville stated, three elements are common to most descriptions of crisis: (a) a threat to the organization, (b) the element of surprise, and (c) a short decision horizon. Crisis planning is about building the capability to identify looming threats to the organization and designing a plan for addressing those threats [11]. It is clear that IS resilience planning and crisis planning overlap considerably: 1 ) they both deal with the future, 2) they both deal with the weaknesses (vulnerabilities) and threats (risks), 3) they both involve creating a plan, and 4) they both involve organizational structures and resources to carry out the plan. However these two planning processes of identifying looming threats and designing a plan are typically carried out in isolation from one another, if they are carried out at all [11].

\section{Research Method}

Critical Realism (CR) based research methodologies provide researchers new prospects to explore complex organizational phenomena in a complete manner.CRbased research can effectively respond to recent calls for improved theorizing and creating IS theories that are systems-oriented and that identify the mechanisms which connect "chains of indeterminate events and complex interactions" [6, p. 45]. This permits researchers to develop and support in-depth causal explanations for the outcomes of specific sociotechnical phenomena.

As formulated by Bhaskar modern critical realism is positioned as an alternative to the positivist and interpretivist paradigms, and influences elements of both to provide new approaches to developing knowledge [16]. Specifically, critical realism acknowledges the role of subjective knowledge of social actors in a given situation as well as the existence of independent structures that constrain and enable these actors to pursue certain actions in a particular setting. As a result, researchers applying methodological approaches consistent with the $\mathrm{CR}$ paradigm are positioned to provide more detailed causal explanations of a given set of phenomena or events in terms of both the actors' interpretations and the structures and mechanisms that interact to produce the outcomes in question.

While critical realism can put up a variety of methodological choices, we focus on the conduct of case study research as the methodology that is perhaps best suited for critical realist studies seeking to develop causal explanations of complex events. This is consistent with our research, as we will answer how senior executives make decisions in time of crisis to ensure IS resilience.

Causality refers to the relationship between an action or thing (cause) and the outcome (effect) it generates. Often, our ability to explain a given phenomenon requires the identification of the factors and relationships which cause it to occur. A primary objective of CR-based research is to provide clear, concise, and empirically supported statements about causation, specifically how and why a phenomenon occurred.

Within CR, causation is not based on regular successions of events or a correlational assessment of event regularities. CR shifts the focus to explicitly describing causality by detailing the means or processes by which events are generated by structures, actions, and contextual conditions involved in a particular setting.

When conducting the Jade Case Study, we followed the dramaturgical model of semi-structured interviewing in qualitative study as proposed by Myers and Newman [17]. The qualitative interview is one of the most important data gathering tools in qualitative research. Rubin and Rubin say that qualitative interviews are like night goggles, "permitting us to see that which is not ordinarily on view and examine that which is looked at but seldom seen" [18]. However, Myers and Newman observed that very few interviewbased IS studies rely on identifiable data generation strategies. In order to address this problem they have prescribed dramaturgical guidelines for conducting qualitative interviews. In this study we have followed this interviewing technique [17].

We started our case study in October 2018 and the project took five months over all. A single researcher, who is a close associate and having had deep access to Jade Software Corporation for more than a decade and who is familiar with the corporate culture, work culture and knows many of the interviewees personally, conducted all the interviews. Jade has formed an IS resilience committee which consists of senior executives and represents various areas within the organization. All members of the IS resilience committee fully participated in the interview process. Data collection was carefully undertaken and a case study database maintained. Each interview lasted an average of one and a half hours and follow-up 
interviews were conducted to eliminate any misinterpretations. Detailed transcriptions of the interviews and resulting summary were shared with participants to ensure that the narrative the researcher captured is consistent with what participants believe they had shared during their interviews and to eliminate any misunderstandings on the part of the researcher. Such confirmation also adds to the credibility of the research.

\section{Jade Software Corporation}

It is crucial in designing case study research to carefully select case study sites. The goal of this study is to understand how senior executives of large organizations make decisions in the context of IS resilience. The focus is on theory building rather than theory testing. Towards this goal, we have selected Jade Software Corporation because it is an exemplar of the theoretical concepts we would expect in the context of IS resilience. Jade Software Corporation Limited was founded in 1978, and is head quartered in Christchurch, New Zealand. Jade brings new digital ideas to life in industries including energy, insurance, agritech, and retail. Thousands of companies around the world rely on Jade every day. Jade is a large organization with 45 major partners, and offices in the United Kingdom, Dunedin, Auckland, Christchurch, Sydney, and Melbourne. Jade experienced a number of challenges because of the Christchurch earthquakes. Jade's primary business operations are located within the disaster zone of the 2010 and 2011 Christchurch earthquakes and as a result, suffered an unsettling blow to business operations. At the time of the adversities, the communications network and electricity cuts were challenging, with personal employee problems resulting in the days after the earthquakes. Jade had in place a fullbodied and prepared IS resilience plan, and had set up special control rooms, as well as establishing a task list and contact tree for emergencies. Therefore, Jade was prepared when the disaster struck. As they were well organized, they quickly adapted to the changed environment and successfully met all contractual requirements throughout the crisis. As all members of the Top Management Team (TMT) at Jade have already experienced a crisis scenario, we expect to observe a full, rich range of IS resilience planning and decision making. Also, we will be able to learn from their experience as to how people learn to adapt and how lessons acquired during the crisis can make a difference later.

The TMT is the link between the board of directors of a firm and the managers entrusted with the day-today functioning of the firm. Consistent with the description, Fama and Jensen have described them as the "apex of the firm's decision control system" [19]. Thus, the TMT is an elite workgroup with a crucial role in the firm's decision-making and face complex, multifaceted tasks that involve both strategic and technical issues. The TMT is responsible for not only decision making but also for implementing and administering those decisions [19]. Jade has a committee that is responsible for risk management and IS resilience planning. The committee consists mostly of members of the executive management team responsible for the various areas of the company. They work together to ensure that all prospective risks are identified, mitigated, and planned for in advance. The TMT's direct involvement and decision making before, during and after the crisis will add realism to this study. As mentioned previously in the definition of IT governance, it is crucial to have the roles and responsibilities defined unambiguously for an effective IT governance framework, which implies the same should be true for IS resilience.

\section{Findings and Discussions}

Jade's IS resilience committee has seven (7) c-suite executives, our text analysis reveals that there are two types of decision makers in the committee - (1) business focused strategic decision makers and (2) technical focused tactical decision makers.

\subsection{Strategy Implementation Bicycle}

Jade's IS resilience committee is made up of members from both business and technical divisions. Members have clearly defined roles to ensure IS resilience at Jade. The business focused strategists of the TMT work in a high and conceptual level predominantly dealing with the IT principles and IT investment and prioritization type decision makings, whereas, the technical focused tactical decision makers of the TMT deal with the IT architecture and IT infrastructure related decision making, and both types play an important role to make decisions related to business application needs.

Interviews of the executives on a broad range of relevant topics indicate a higher degree of agreement rather than disagreement between the two decision types, so we can say that they are functioning more as a team rather than as individuals. Eight key factors essential for successful IS resilience implementation during the analysis. All of the kappa coefficients were evaluated using the guideline outlined by Landis and Koch, where the strength of the kappa coefficients $=0.01-0.20$ slight; 
0.21-0.40 fair; 0.41-0.60 moderate; 0.61-0.80 substantial; 0.81-1.00 almost perfect. In our case overall percent agreement was $92.86 \%$ and kappa $=0.86$, which signifies substantial inter-rater or observer consistency. [20].

The split between strategy and implementation is very crucial for Jade to make the right decisions which can be explained through the "Strategy-Implementation" bicycle. This bicycle model will be helpful to visualize at a high and conceptual level the split and relationship between the strategy and implementation cycles of IS resilience planning. implemented and monitored in the implementation cycle. As a result of continuous evaluation, critical services may continue without any changes or may need to be innovated and re-enter the strategy cycle through a new critical service. This helps decision makers at Jade to identify critical services early, evaluate potential solutions, and then implement them.

As exemplified during interviews, "key risks are identified and understood and then we deal with them [risks]." Another executive stated, "we identify the key services first and then walk backwards to facilitate those services. This way a transformation happens from

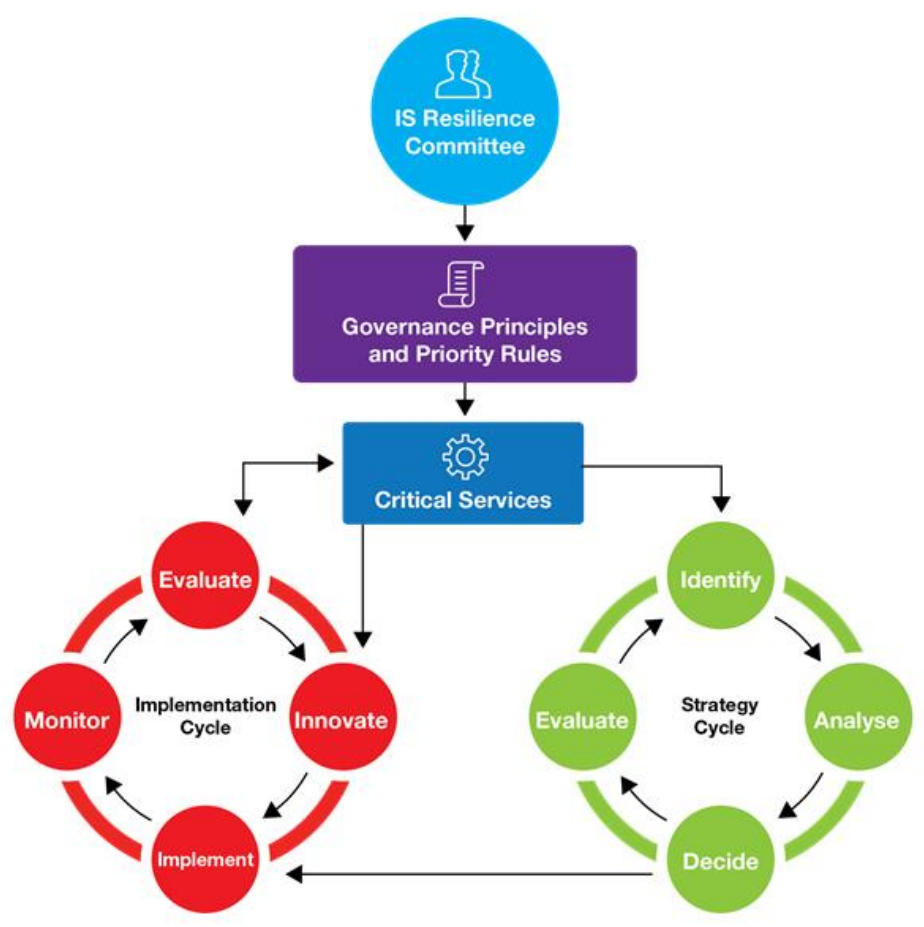

\section{Figure 1: Strategy-Implementation "Bicycle" at Jade Software Corporation}

As shown in figure 1, the IS resilience committee based on the business/IT strategy, drives the definition and application of the IT governance principles and priority rules and then defines the critical services. The committee identifies the critical services and relates them to business needs and specifies both service owners and consumers to impose accountability and ensure smooth and uninterrupted delivery of service. The approved critical services are managed in the strategy cycle. After a decision has been made, critical services need to be implemented so they become part of the implementation cycle. These decisions are then 'passionate drive from individuals' to 'service critical thinking." The momentum generated due to this bicycle model in decision making shows that IS resilience plans are never parked at Jade but are living documents. This has been described and emphasized eloquently by several committee members; "In times of crisis, plans go out of the window, it is important not to park those plans". "Planning is critical but continual review is important." "We had a plan and people knew what to do." This strategy-implementation bicycle, derived from their interviews as represented in Figure 3, has been verified and validated by the senior executives at Jade. 
IT governance has been defined as the accountability framework for IT decisions to enable desirable behaviors [5] and is viewed as a key responsibility of top management [14]. The design of an organization's IT governance framework is recognized in the literature as involving key trade-off decisions. For example, when IT decision rights are exclusively allocated to an IT unit, there is a considerable risk that the business interests are not adequately considered, resulting in a lack of business/IT alignment [14]. On the other hand, if IT decision rights are allocated to business units, considerations of a technical nature, and considerations from an enterprise-wide perspective are not sufficiently addressed. The Strategy-Implementation cycle at Jade is perfectly aligned to the IT governance framework, so much that it is functionally integrated with IT governance; in other words, IS resilience planning is one of many aspects of organizational and IT governance at Jade.

\subsection{Causal Model of IS Resilience}

We will discuss causal perspective to explore the decision making of two different types of decision makers at Jade. The important message here is that the model combines various subjective and objective factors derived from careful reflection. Figure 2 depicts a causal model of IS resilience with trigger events, control events, risk events, mitigate events and consequence events. The causal model has been used to explain how decisions are made and prioritized by the TMT at
Jade. This is a major contribution of our study as it explains the "gut-feel" decisions, which are based on doing all the reasoning "in the head" of the decision makers or relying on intuition. The causal model helps us to explore "what lies under the bonnet" of the TMT decision motivation. The causal model comprises: 1) the event itself, 2) at least one consequence event that characterizes the impact, 3) one or more trigger events, 4) one or more control events which may stop the trigger event from causing the risk event, and 5) one or more mitigating events which help avoid the consequence event.

With this causal perspective, our risk event is "compromised by IS resilience", which may be triggered by any form of adverse circumstance. The risk event also has a number of possible outcomes or consequences. Multiple controls can be put in place to avoid risk events and in case the risk event takes place then there are multiple mitigants that will reduce the impact of consequences. We found that the ability to decompose an IS resilience issue into chains of interrelated events should make decision making more meaningful, rational, practical and coherent.

The causal model clearly shows that the consequences can be divided into two types according to Weill's IT governance framework, hence two types of decision maker in the TMT at Jade, the business and technical strategists, who complement each other to ensure IS resilience. As explained during the interview, "[The] IS resilience committee needs wide-spread knowledge, it is so complex that no one person understands it.

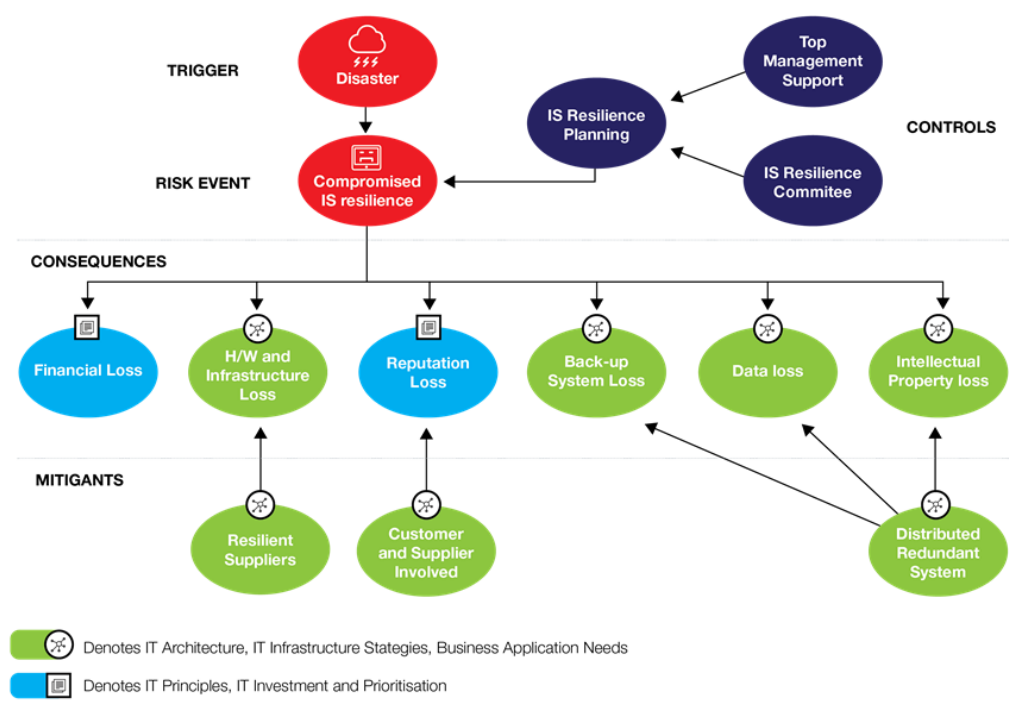

Figure 2: Causal Model for IS 
We formed a collaborative team of members with different expertise. We have identified that not only having a plan is critical but execution of the plan is equally important. As a collaborative effort the committee first identified key risks. In order to derive those risks we looked at the service level agreements and customer contracts, then we have done a thorough business impact analysis, and have graded customer contracts and SLAs to address various business impacts."

IS resilience planning requires both strategy formation and execution. Jade TMT rejects firmly the notion that they are separable and rather consider them as a closely bonded pair. They view strategy as a continuous process involving decisions and actions, not a periodic process involving only decisions. Two important lessons were learnt as a result.

IS Resilience Planning Process and Implementation: rather than a rigid hierarchy of plans derived from an 'event-based' model, it is critical to have a more flexible plan based "service-recovery", which is neither scenario based nor event specific. However, the context in which IS resilience plans are implemented are by definition highly uncertain, ambiguous, laden with risk, and require employees at all levels of the firm to act with greater degrees of autonomy and discretion so as to remain flexible in adverse circumstances or times of crisis. As highlighted by the senior executives, "In time of crisis plans go out of the window, it is important not to park those plans". This makes good sense in the unique context of IS resilience planning.

Resilience Strategy: clear strategy aligned to organizational goals and priorities must be formulated which has to be embedded in the organization's culture. Executives at Jade not only value data-driven quantifiable decision making but also strongly believes that the organizational culture plays an important role in IS resilience.

Sincere Top Management Commitment to Resilience: a vital requirement to IS resilience planning is the commitment at top management level and to reach effective IT governance, two-way communication and a good participation/collaboration relationship between the business and IT people are desirable. Adequate financial support to implement is also very important.

It is evident from the interviews that Jade values their people and put them in the core of their success. According to the executives, organization culture and human capital along with data and measurement are of immense importance to formulate a successful IS Resilience strategy, it infuses every aspects of organizational strategy, from prioritization and goalsetting to strategy formulation through resource allocation and day-to-day execution. This also reveals another very important assumption, that markets are composed of real human beings rather than 'rational economic agents'. Real people are capable to show passion, benevolence, insight, intellect, innovation and perseverance. They are more impressive than economic agents as they exhibit moral and ethical values, altruism, trust, compassion, reciprocity, justice, loyalty and love. Educating and Knowledge Sharing: resilience includes learning and knowledge sharing, adaptation, innovation and staff training. Managers and employees need to be educated on a regular basis to create an organization wide resilience culture. As identified by Kayes, "It is the 'experienced' [person] who knows the limitations of all anticipation, the insecurity of all human plans. Experience teaches the incompleteness of all plans [2]." This establishes a deep connection between resilience and learning, and points to a style of learning orientation that is closely aligned with resilience. It is also consistent with the findings about the need for a flexible plan, since training and education are necessary, if employees at all levels of the firm will be expected to act with greater degrees of autonomy and discretion in times of crisis. In this case, therefore, training and education become a vehicle for the transference of riskbearing and decision rights to employees at all levels of the firm.

In case of IS resilience planning, where the environment is unstable and performance function is uncertain, executives can reduce uncertainty by gathering information from different sources and observing the effects of resource allocation. As learnt from Jade, this component of successful IS resilience planning can be summarized as:

Continuous Testing and Monitoring: conducting dryrun or live test scenarios for testing specific service recovery strategies and regularly re-assessing risks and mitigation strategy. This finding also follows our finding about training and education, since it serves a purpose to enable employee preparedness at all levels of the firm.

Regular and Transparent Communication: wellplanned communication and change management is essential to effectively adapt to turbulent changes.

Choose Your Partners Wisely: focus on key resilience attributes that really matter while choosing your partners is essential. This is also important while migrating to cloud environment.

Strong Understanding of Value Chain: important message is "connectedness", value chain takes into consideration different types of inter organizational relationships, such as, suppliers, customers or the government. 


\section{Conclusion}

The allocation of information technology (IT) decision rights between IT units and business units remains an important IT governance challenge. Companies that do not design an appropriate accountability framework for IT run the risk of business losses due to poor management decisions and misaligned IT priorities. While more detailed empirical work is necessary to elaborate and confirm the bicycle model, it is believed that a useful starting point has been made. Understanding the decision making by senior executives to ensure IS resilience will inform us to develop an IS resilience framework that encompass IT governance structures, processes and relational mechanisms. Effective IS resilience does not happen accidentally, rather requires thoughtful planning. We have described IS resilience planning in light of a strategy-implementation bicycle and causal model to understand decision makers' perspective to understand decision priorities. There are a number of avenues of future research, including examining a greater range of organizations. Future empirical research should attempt to understand the IS resilience decision priorities and characteristics of resilient organizations. Finally, results have implications both for researchers who are looking for theories that explain the importance of IS resilience and business managers and owners who are challenged with decisions about how to design resilient information system framework for their organization. This study contributes to the existing literature from both a theoretical viewpoint and a practical viewpoint.

\section{References}

[1] Maurer, F., and Lechner, U, "From Disaster Response Planning to e-Resilience: A Literature Review," BLED 2014 Proceedings, 2014, Paper 32.

[2] Kayes, D.C, Organizational Resilience: How Learning Sustains Organizations in Crisis, Disaster, and Breakdowns, New York: Oxford University Press, 2015.

[3] Earl, M. J, "Experiences in Strategic Information Systems Planning," MIS Quarterly, March, 1993, pp. 1-27.

[4] Hann, J and Weber, R, "Information Systems Planning: A Model and Empirical Tests", Management Science (42: 7), 1996, pp. 1043-1064.
[5] Weill, P. and Ross, J.W, IT governance: How Top Performers Manage IT Decision Rights for Superior Results, Watertown, MA: Harvard Business School Press, 2004.

[6] Grover, V., Henry, R.M., and Thatcher, J.B, "Fix ITbusiness relationships through better decision rights", Communications of the ACM, 50(12), 2007, pp.80-86.

[7] Flyvbjerg, B, "Five Misunderstandings About Case-Study Research”, Qualitative Inquiry, 2006, pp. 219-245.

[8] Kuhn, T. S. (1987). What are scientific revolutions? In L. Kruger, L. J. Daston, \& M. Heidelberger (Eds.), The probabilistic revolution, Vol. 1: Ideas in history, Cambridge,MA: MIT Press, 1987, pp. 7-22.

[9] Yin, R. K, The Case Study Anthology, Thousand Oaks, CA, Sage, 2004.

[10] Muller, G., Koslowski, T., and Accorsi, R, "Resilience A New Research Filed in Business Information Systems," ACM Symposium on Business Computing, 55(4), 2013, pp. 112.

[11] Vargo, J., and Seville, E, "Crisis strategic planning for SMEs: finding the silver lining," International Journal of Production Research, 7(3), 2011, pp. 5619-5635.

[12] Heeks, Richard and Ospina, Angelica V, "Conceptualising the link between information systems and resilience: A developing country field study", Information Systems Journal, published online first 19 January 2018.

[13] McManus, S., Seville, E., Vargo, J., and Brunsdon, D, "Facilitated Process for Improving Organizational Resilience," Natural Hazards Review, 3(4), 2008, pp. 81-90.

[14] Haes, S. D., and Grembergen, V., W, "IT governance and its mechanisms", Information Systems Control Journal, 1(1), 2004, pp. 27-33.

[15] Jensen,M.C. and Meckling, W.H, Foundations of Organisational Strategy, Lars Werin and Hans Wijkander, eds., Blackwell, Oxford, 1992.

[16] Bhaskar, R.A. et al, The formation of critical realism: a personal perspective. London ; New York: Routledge, 2008.

[17] Myers, M. D., \& Newman, M, The qualitative interview in IS research: Examining the craft. Information and Organization, 17(1), 2007, pp. 2-26.

[18] Rubin, H. J., \& Rubin, I. S, Qualitative interviewing: The art of hearing data (2nd ed.). Thousand Oaks, CA: Sage, 2005. [19] Fama, E. F., and Jensen, M. C, "Separation of Ownership and Control," Journal of Law and Economics, 1983, pp. 1-30. [20] Landis, J. R., \& Koch, G. G, The measurement of observer agreement for categorical data. Biometrics, 33, 1977 , pp. $159-174$ 